\title{
OUTCOMES-BASED ASSESSMENT AS AN ASSURANCE EDUCATION TOOL
}

\author{
Susan Older and Shiu-Kai Chin \\ Department of Electrical Engineering and Computer Science, Systems Assurance Institute, \\ Syracuse University, Syracuse, NY 13244
}

\begin{abstract}
The goal of Syracuse University's Certificate of Advanced Study in Systems Assurance (CASSA) program is to develop students who (1) comprehend the concepts underlying security and system assurance; (2) can apply those concepts to construct assured systems; and (3) can critically analyze and evaluate systems' conformance to their requirements. Because of this third requirement, a key component of the CASSA program is an emphasis on using formal mathematics and logic to provide a rigorous basis for the assurance of information and information systems.

Our purpose in writing this paper is twofold. The first is to report on our progress in delivering an assurance curriculum with a strong emphasis on logic and formal methods. Specifically, we describe what we are teaching in two of our foundational courses, as well as what our students are learning. The second and broader purpose is to advocate the use of an outcome-based approach when developing IA courses and curricula. We have found that focusing on the desired educational outcomes from the outset has made it easier to identify what is working and what is not, and we wish to share our experiences.
\end{abstract}

Key words: Formal methods, educational outcomes, assessment of student learning, assurance.

\section{INTRODUCTION}

Systems Assurance focuses on the correctness, integrity, reliability and security of the systems upon which our society is constructed and thus depends. The multi-disciplinary Systems Assurance Institute (SAI) at Syracuse University has been established to promote improvement in systems assurance through research, education, and technology transfer. The 
SAI comprises faculty from four distinct colleges: the College of Engineering and Computer Science, the School of Information Studies, the Maxwell School of Citizenship and Public Affairs, and the Newhouse School of Public Communications. The model that we have adopted for educational programs allows each academic unit to define its own disciplinespecific foundation, while taking advantage of other units' offerings for electives to produce well-rounded students.

The most mature of these individual programs is the Master's-level Certificate of Advanced Study in Systems Assurance (CASSA), which has been approved by the New York State Department of Education. The CASSA program exists within the framework of the Computer Science and Computer Engineering Master's programs: students must satisfy all requirements of their home Master's program, as well as satisfying CASSAspecific requirements. A key-and, we believe, unique-component of the CASSA program is its emphasis on using formal mathematics and logic to provide a rigorous basis for the assurance of information and information systems. All students must take a combination of courses that provides hands-on experience not only in systems building but also in using formal methods to analyze and evaluate system behavior.

Our purpose in writing this paper is twofold. The first is to report on our progress in delivering an IA curriculum with a strong emphasis on logic and formal methods. In [OC02], we described our experiences in developing the CASSA program, as well as the challenges inherent in incorporating mathematical and logical rigor into an IA curriculum. In this paper, we provide more specific information about what we are doing in our courses, as well as our ongoing attempts to answer the following questions:

\section{To what degree are we being successful? What are our students learning?}

We discuss two specific courses that serve as elective courses in the CASSA program: Modeling Concurrent Systems (CIS 632) and Principles of Network Security (CSE 774). For each course, we describe the educational objectives, what we have done to realize those objectives, our observations of student achievement (both subjective and objective), and the changes we have made or intend to make as a result of these observations. We hope that our experiences are informative for others interested in introducing formal methods and logical rigor into an IA curriculum.

The second and broader purpose is to advocate the use of an outcomebased approach when developing IA courses and curricula. To be explicit, we do not wish to promote additional bureaucracy or the complex instrumentation of courses. Rather, we have found that focusing on the desired educational outcomes from the outset has made it easier to identify 
what is working and what is not, and we wish to share our experiences. In our opinion, this approach is especially useful for developing IA curricula. IA is such a broad field that no individual program can cover everything. Emphasis on desired outcomes helps identify which topics to include and in what depth. Furthermore, the greater specificity of desired outcomes allows for more detailed and precise discussions across disciplines.

The rest of this paper proceeds as follows. In Section 2, we describe the high-level educational outcomes of our CASSA program and how the requirements relate to these outcomes. The next two sections then illustrate how one of the high-level CASSA outcomes is addressed through two separate courses. Section 3 presents the course Modeling Concurrent Systems, an applied concurrency-theory course in which IA applications are an integral component. Section 4 focuses on Principles of Network Security: this course uses a variety of logical systems to describe, verify, and analyze properties related to network security. We conclude in Section 5 with some final observations.

\section{CASSA EDUCATIONAL OUTCOMES}

In 1998, the EECS Department adopted an outcome-based curricula design approach [Syr98], in which we first formulate the outcomes we desire of our graduates and then use those outcomes to guide the development of rational curricula. This approach represents a distinct shift from the traditional faculty-centered viewpoint of "what do we teach?" to a studentcentered viewpoint of "what do our students learn?"

We made use of this approach in developing the CASSA program, focusing on our expectations for those students who successfully complete our program. No single curriculum can possibly address all of Information Assurance: concentrating on the desired educational outcomes helped us determine how to structure our program. In our case, the goal was to develop a coherent collection of courses to ensure the following educational outcomes:

1. Students comprehend the concepts underlying security and systems assurance.

2. Students can apply those concepts to construct assured systems.

3. Students can critically analyze and evaluate systems' conformance to their requirements. 
Not surprisingly, these outcomes reflect EECS department's long-standing emphasis on the use of mathematical and logical methods in engineering, computer science, and security.

The CASSA educational outcomes are broadly addressed by the program requirements. For example, students must successfully complete both the Systems Assurance Seminar and a non-technical IA elective course (such as telecommunications policy, Internet law, or e-commerce). The seminar course serves as a gateway to more advanced assurance courses, introducing basic terminology and many of the nonmathematical issues related to information assurance. Together, these two courses are intended to address Outcome 1. Furthermore, students must successfully complete a total of five courses from the Foundations for Assurance and Assurance Applications tracks, including at least two courses from each track. The Foundations track includes courses in cryptography, concurrency theory, and logical principles of network security. The Applications track includes courses such as Internet security, computer security, and security of wireless networks. Via this requirement, students are assured to receive hands-on experience both in building systems and in using formal methods, thus addressing Outcomes 2 and 3.

This setup raises an obvious question: what specifically can students do in the areas of constructing assured systems and critically analyzing and evaluating systems' conformance to their requirements? In this paper, we focus on the formal-methods aspect and describe our experiences assessing our students' learning in two separate Foundations for Assurance courses. We realize that, when the topics of outcomes and assessment are initially brought up, the typical reaction is resistance, due to a belief that it will be cumbersome, bureaucratic, and of little practical use. This has not been our experience. We have found that a good way to start is to follow Diamond's suggestion ([Dia98], page 134):

As an alternative to writing objectives in the abstract, ... [one can]

develop strong, clear objectives by playing the role of the student and asking, "If I'm your student, what do I have to do to convince you that I'm where you want me to be at the end of this lesson, unit, or course?"

This approach is exemplified by the description of Modeling Concurrent Systems in Section 3. One can also take a more structured approach suggested by Diamond and write objectives that include "a verb that describes an observable action" and "a description of the conditions under which the action takes place: 'when given $\$ \mathrm{x} \$$, you will be able to ...' " ([Dia98], page 132). This approach is used in Principles of Network Security, which is described in Section 4. 


\section{CIS 632: MODELING CONCURRENT SYSTEMS}

The purpose of this course is to provide students with an in-depth understanding of the process-algebraic approach for specifying, modeling, and analyzing system behavior. Process algebras such as CSP [Hoa85, Ros98] and CCS [Mil80] provide a way to describe system behavior in terms of the events (i.e., abstract actions deemed "observable") that can occur. The underlying theory also includes several useful notions of program equivalence and refinement, which are useful for compositional reasoning and analysis. There are automated and semi-automated tools available that allow one to apply the theory in practice to verify properties of nontrivial applications.

In the last two offerings, this course has been taught as a $400 / 600$-level split: undergraduate and graduate students attend the same lectures, but they have different assignments, exams, and grading criteria. In Fall 2002, thirteen students (all undergraduate computer-science majors) took the CIS 400 version, while eleven graduate students (from both computer science and computer engineering) took CIS 632. The following discussion and statistics refer only to the graduate version of the course, except for where undergraduates are explicitly mentioned.

\subsection{Educational Outcomes and Course Content}

In Fall 2002, we focused on the use of CSP to specify and verify concurrent systems and to understand the emergent behavior of such systems. The educational outcomes appear in Table 1.

Table 1. CIS 632 Educational Outcomes

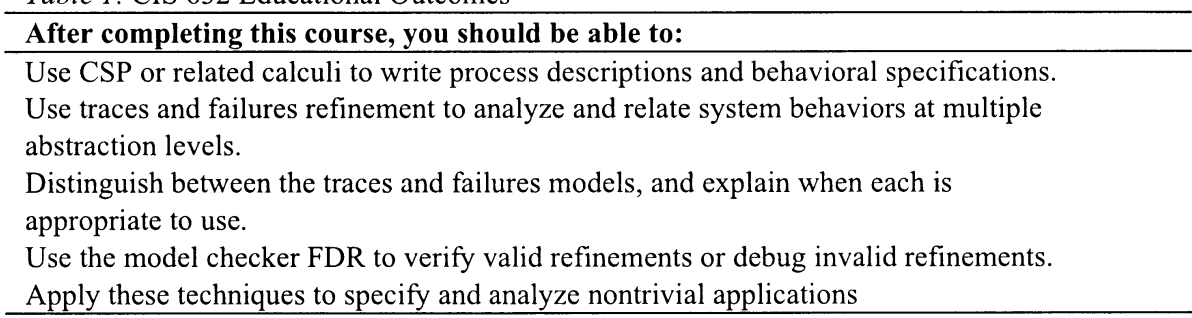

We used Steve Schneider's CSP textbook [Sch00], supplemented with several exercises and examples from Bill Roscoe's textbook [Ros98]. In the first portion of the semester, we introduce the basic operators of CSP, their operational semantics, and the trace and failures models, both of which induce notions of refinement. The trace model, for example, formally describes a process's behavior in terms of the set of traces-that is, 
sequences of events-that it can perform. These trace sets provide a basis for comparing, equating, and refining processes. Two processes are traceequivalent when they have precisely the same sets of traces. A process $Q$ refines $P$ in the trace model (written $P \leq_{T} Q$ ) if every trace of $Q$ is also a trace of $P$. Intuitively, if $P$ corresponds to a specification of permissible behavior and $Q$ refines $P$, then $Q$ is guaranteed to exhibit only permissible behaviors.

Similarly, the failures model supports a notion of refinement based on a process's set of failures, which pair traces with sets of events. A process has the failure $(t, X)$ if it can perform the trace $t$ and then reach a state where its only possible actions involve events not in the set $X$. The failures model provides a finer notion of equivalence and refinement than the trace model does: it distinguishes processes not only by what they are able to do but also by what they are able to refuse to do. As a result, it supports reasoning about the potential for deadlock. A process $Q$ refines $P$ in the failures model (written $P \leq_{S F} Q$ ) if $P \leq_{T} Q$ and every failure of $Q$ is also a failure of $P$.

We also introduced the model checker FDR2 [For97], which provides automated support for applying these notions in practice. The advantage of using a model checker is that one can analyze much larger systems than is possible by hand. In addition, model checkers can be useful in debugging designs: for example, when a desired refinement $P \leq_{T} Q$ fails, FDR2 produces a witness (i.e., a trace of $Q$ that is not allowed by the specification $P)$.

The final third of the course is spent on using CSP and FDR2 to analyze several different problems and protocols, such as the alternating-bit protocol and a distributed-database cache-coherency protocol. For three class periods, students worked in small groups of 4-5 students to specify and implement a one-lane bridge that safely supported bi-directional traffic. We also spent two lectures on the Needham-Schroeder key-exchange protocol and Gavin Lowe's use of CSP and FDR2 to uncover a previously unknown flaw in it [Low96]. This analysis uses CSP to model the system as the parallel composition of an initiator, responder, and intruder; CSP is also used to describe the desired authentication properties. FDR2 can then be used to try to validate refinement relationships between the system and the two desired properties. The result of FDR2's analysis is a witness trace that highlights a possible man-in-the-middle attack.

\subsection{Assessment Methods}

Grades for CIS 632 were based on an equally weighted combination of homework assignments, quizzes, a final exam, and a final project. The purpose of homeworks was to keep students up-to-date with the material 
discussed in class and to familiarize them with the tools. The homeworks also served as simple formative-assessment tools: if several students had questions while working on the assignments or did poorly on particular questions, there was indication that certain topics needed to be reviewed again.

In total, there were six homeworks. The first two homeworks concentrated on sequential processes. The first homework required students to write CSP processes for a variety of scenarios, including one of their own choosing. For the second homework, students wrote machine-readable CSP and used the process animator ProBE [For98] to interact with their processes and to test their understanding of the CSP transition rules. The third and fourth homeworks focused on the various parallel operators of CSP: the third homework assessed basic understanding of the operators, while the fourth homework challenged the students to use the operators to introduce constraints on a system. The final two homeworks assessed students' understanding of the primary abstraction operators (i.e., hiding and renaming), as well as their mastery of the traces and failures models.

The quizzes and the final exam-all closed book and closed notes-served as summative-assessment tools, letting students demonstrate their understanding of the fundamental concepts. The quizzes were relatively lightweight and intended primarily as sanity checks. Typically, $60-75 \%$ of a quiz's points were for basic understanding of fundamentals (e.g., drawing transition graphs of processes, identifying a process's set of traces or failures, determining simple refinement relationships between processes). The remaining points tested students' deeper understanding of the concepts, such as writing a CSP process to model a scenario, validating or refuting claims about refinement relationships, and generating CSP processes that have (or fail to have) certain properties. The final exam placed more weight on these latter sort of questions: 34 points (out of 100) were for fundamentals, 30 were for writing CSP to model a scenario, and 36 were for validating/refuting a variety of claims about refinement relationships. Two examples of these claims follow:

- If $f(P) \leq_{S F} f(Q)$ and $f$ is a renaming function, then $P \leq_{S F} Q$.

- For all processes $P, Q$, and $R$, and for all sets $X$ and $Y$, the following are trace equivalent:

$$
\left.P\|\|_{X} Q \|_{Y} R\right) \text { and }\left(P \|_{X} Q\right) \|_{Y} R \text {. }
$$


The first claim states that every failures-refinement relationship that holds between renamed processes (the process $f(P)$ behaves like $P$, except for a renaming of events via the function $f$ ) must also hold between the original processes. The second claim states that distinct parallel-composition operators are necessarily associative with one another. Both claims happen to be false.

The final project required students to use machine-readable CSP and FDR to model and reason about a nontrivial system or protocol. Students were required to submit annotated machine-readable CSP scripts containing descriptions of their system and of the desired behavioral properties, as well as the assertions (e.g., refinement or deadlock checks) necessary for validating those properties. Students were also required to submit 8-10 page project reports containing the following features: a high-level description of both the problem they were solving/analyzing and their CSP solution; a description of their use of refinements (e.g., explaining why trace refinements were used instead of failures refinements, or vice versa); and some analysis of their experience (e.g., unexpected results or design choices that were particularly good or bad). This paper was graded for grammar, spelling, and style, as well as for content. Students analyzed a variety of protocols and algorithms, including the Bully election algorithm, the Needham-Schroeder and TMN security protocols, the two-phase commit protocol for distributed transactions, and a distributed-sum algorithm.

\subsection{Observations}

We have found it useful to introduce the automated tools early in the semester: it helps students view CSP more like a programming language (and therefore "real") than just a mathematical notation. While grading homeworks early in the semester, we noticed that the undergraduates seemed to write higher quality CSP code: the graduate students wrote code that was technically correct, but it had a very strong imperative flavor (e.g., large numbers of nested conditionals). We postulate that, because the undergraduates all had functional-programming experience, they were better prepared to think about computational solutions in a non-imperative way. ${ }^{3}$

We have also found that students at both the graduate and undergraduate levels have difficulty grasping the distinction between the properties one wishes to prove about a system and the constraints imposed upon a system through parallel composition. The properties are described as CSP processes,

3 A student from the Fall 2001 class made a similar observation a few weeks later in an unsolicited comment to the CIS 632 instructor. The student was using Scheme in another class, and he suggested that functional programming should be a prerequisite for CIS 632 . 
and one can check whether the system satisfies those properties by checking whether a refinement relationship exists. Constraints are also represented by CSP processes, placed in parallel with the system to enforce certain properties. The confusion seems to arise because the same language is used both to express properties and to describe the system design: students have difficulty maintaining that distinction.

On the CIS 632 final exam, students had very little difficulty with drawing transition graphs or identifying a process's traces and failures. The average grade (out of 10 students) for the combination of these questions was $85 \%$, with a high mark of $100 \%$ (two students), a low of $62 \%$, three students between $74 \%$ and $77 \%$, and the rest between $85 \%$ and $97 \%$. Students were also asked to write a CSP process to describe a banking account that allowed deposits, withdrawals (with limited overdraft protection), and queries, as well as requests to change the overdraft limit. Students then had to add a parallel constraint to the system to limit the number of rejected overdraft-change requests. On this question, the average grade was $74 \%$, with a high of $93 \%$ and a low of $53 \%$; five students received between $60 \%$ and $73 \%$, and three received between $87 \%$ and $90 \%$. The final question required students to judge the validity of various claims and to provide convincing explanations for their answers. Students had much more difficulty with this question: most students correctly identified the truth or falsity of the claims (worth a third of the points) but provided insufficiently convincing explanations. Here, the average was $63 \%$, with a high of $89 \%$ (two students) and a low of $36 \%$; the remaining students received grades between $39 \%$ and $86 \%$, in a fairly even distribution.

\subsection{Adjustments}

The results from the final exam suggest that students have good intuition about the concepts but need more practice in justifying answers rigorously: for example, many students would give a specific example to justify that a general claim was true. We plan to structure homeworks and lectures to give students more practice in providing rigorous and logical justifications for answers, as well as to review common logical fallacies.

The confusion between constraints and properties has triggered changes in two successive years. Having identified the problem in Fall 2001, we made a concerted effort in Fall 2002 to mediate it. In the bridge project, two groups were charged with identifying the desirable properties and describing them in CSP; the remaining groups were to model the bridge and a trafficlight system in accordance with a proposed solution. We would then, as a class, combine both parts in a single file and check the necessary refinement relationships. The expectation was that, by separating the tasks, students 
would concentrate on either properties or the system and be able to see how they relate to each other. However, both groups charged with writing properties repeatedly asked for clarification of their task: they had trouble comprehending how their properties were related to the overall system.

We plan to use the process algebra CCS in the next offering of this course. The CCS notion of synchronization differs from the CSP notion in a way that avoids the constraints idiom. Furthermore, the available automated tools for CCS (e.g., the Concurrency Workbench [CPS93]) support the use of temporal logic for describing desirable properties. We believe that the use of different notations will help students distinguish between system-level descriptions and their behavioral properties.

\section{CSE 774: PRINCIPLES OF NETWORK SECURITY}

Principles of Network Security is an analytical course that uses predicate calculus, higher-order logic, and specialized logical systems to describe, specify, and verify the correctness and security properties of network security protocols, algorithms, and implementations. Students use formal logic to rigorously analyze cryptographic algorithms, key-distribution protocols, delegation, access control, electronic mail, and networks of certification authorities.

This course is open only to graduate students. Because this course is fairly novel even among IA curricula and has no standard textbook, we describe its contents in more detail than the course in the previous section.

\subsection{Educational Outcomes and Course Outline}

Both the educational outcomes and our discussion about this course make use of a modified Bloom's taxonomy [Blo74] to classify and distinguish the many kinds of knowledge and abilities. Some kinds of knowledge are at relatively low levels (e.g., recalling that $15_{16}=21_{10}$ ), while others are at very high levels (e.g., evaluating whether an implementation meets a specification and requirement). The outcomes, which appear in the course's online syllabus, are listed in Table 2.

The first part of the course (outlined in Table 3) deals with network security fundamentals and covers the standard topics: basic security properties, cryptographic algorithms (e.g., DES and RSA), authentication and hash functions, digital signatures, protocols and certificates. The primary reference is William Stallings' classic text [Sta99]. 
Table 2. CSE 774 Educational Outcomes

\author{
Comprehension \\ Define the meaning of security services such as confidentiality, integrity, non-repudiation, \\ and authentication \\ Describe the characteristics of private key and secret key cryptographic systems \\ Describe cryptographic algorithms for encryption, hashing, and signing \\ Describe cryptographic protocols for session-based security such as Kerberos, and store-and- \\ forward security such as Privacy Enhanced Mail \\ Describe basic principles of trust topologies and networks of certification authorities
}

\section{Application}

When given a block diagram or functional description of an implementation, you should be able to represent the implementation using predicate calculus

When given protocol descriptions and trust hierarchies, you should be able to use specialized security calculi such as the logic of authentication for distributed systems to describe the protocol and trust relationships

When given a trust topology, determine the necessary certificates for establishing trust in a key
\end{abstract}

\section{Analysis}

When given a set of assumptions and a goal to prove, you should be able to prove, using formal inference rules, if the security goal is true or not

When given a set of certificates, you should be able to formally derive whether a key is associated with a particular principal
}

\title{
Synthesis \\ When given a description of a system or component and its specification and security properties, you should be able to construct a theory that describes both, and show if the security properties are supported \\ When given a description of a trust topology, you should be able to create a formal description of certificates and trust relationships for the certification authorities
}

\section{Evaluation}

When given a theory, inference rules, and a proof, you should be able to judge if the proof is correct

When given a specification and implementation, you should be able to judge whether the implementation satisfies its specification

Table 3. CSE 774 Topics in Security Fundamentals

\begin{tabular}{ll}
\hline Topic & Primary References \\
\hline $\begin{array}{l}\text { Basic Security Properties: confidentiality, } \\
\text { authentication, integrity, non-repudiation, }\end{array}$ & [Sta99] $\S 1$ \\
$\begin{array}{l}\text { access control, availability; mechanisms; } \\
\text { attacks }\end{array}$ & \\
$\begin{array}{l}\text { Conventional Encryption: DES, Electronic } \\
\text { Code Book, Cipher Block Chaining }\end{array}$ & {$[$ Sta99] $\S 3.1-\S 3.3, \S 3.7$} \\
$\begin{array}{l}\text { Confidentiality: placement of encryption, } \\
\text { traffic confidentiality, hey distribution }\end{array}$ & [Sta99] $\S 5.1-\S 5.3$
\end{tabular}




\begin{tabular}{ll}
\hline Topic & Primary References \\
\hline $\begin{array}{l}\text { Public-key Cryptography: principles of } \\
\text { public-key cryptosystems, RSA, key }\end{array}$ & [Sta99] $6.1-\S 6.3$ \\
management & \\
$\begin{array}{l}\text { Message Authentication and Hash } \\
\text { Functions: authentication requirements, } \\
\text { authentication functions, message }\end{array}$ & [Sta99] $88.1-\S 8.4$ \\
$\begin{array}{l}\text { authentication codes, hash functions } \\
\text { Hash Functions: Secure Hash Algorithm }\end{array}$ & \\
$\begin{array}{l}\text { (SHA-1) } \\
\text { Digital Signatures and Authentication } \\
\text { Protocols: digital signatures, authentication } \\
\text { protocols, digital signature standard } \\
\text { Authentication Applications: Kerberos, } \\
\text { X.509 authentication service }\end{array}$ & [Sta99] $\$ 10.1-\S 10.3$ \\
\hline
\end{tabular}

The second part of the course (see Table 4) deals with reasoning about freshness of protocols, replay attacks, and role-based access control. We use the BAN logic [BAN90] to reason about freshness and potential replay attacks. We also introduce the formal definitions and properties of role-based access control (RBAC), as described by Ferraiolo, Sandhu, and Kuhn in [FBK99, FSG+00] and [FK92]. The knowledge expected of students is at the levels of comprehension, application, analysis and synthesis. Specifically, when given informal descriptions of key-exchange protocols, students are expected to be able to describe the protocol abstractly in the BAN logic; postulate initial beliefs about key associations, scope of authority, freshness of nonces, and so on; and show that, at the end of the protocol, belief in the distributed keys has been established. A similar set of skills is expected related to RBAC: when given an organizational structure of roles, students are expected to write down role-containment relations and derive the membership relations that are implied by a specific organizational structure.

Table 4. CSE 774 Topics in Belief Logics and Role-Based Access Control

\begin{tabular}{ll}
\hline Topic & Primary Reference \\
\hline BAN Logic & [BAN90] \\
& [FBK99] \\
RBAC Definitions and Properties & Also: [FSG+00], [FK92] \\
\hline
\end{tabular}

The third part of the course (outlined in Table 5) focuses on authentication, delegation, and access control in distributed systems. Nine weeks is spent on this topic, the major focus of the course. The technical content of this part centers on the Abadi calculus [ABLP93, LABW92] for reasoning about principals, their statements, and their beliefs. 
Table 5. CSE 774 Topics in Authentication, Delegation, and Access Control

\begin{tabular}{|c|c|}
\hline Topic & Primary References \\
\hline \multirow[t]{2}{*}{ Underlying Semantics and Model } & [HK00] $\S 1-\S 3$ \\
\hline & Also: [ABLP93] $\S 3.3-\S 3.4$ \\
\hline \multirow[t]{2}{*}{ Axioms for Principals and Statements } & [LABW92] $\S 3,[\mathrm{HK} 00] \S 4.1-\S 4.3$ \\
\hline & Also: [ABLP93] $\S 3.1-\S 3.2$ \\
\hline Channels and Encryption & [LABW92] §4 \\
\hline Group Names & [LABW92] $\S 5.3$ \\
\hline \multirow[t]{2}{*}{ Roles and Programs } & [LABW92] $\S 6$ \\
\hline & Also: [HK00] $\S 4.4-\S 4.5$ \\
\hline \multirow[t]{2}{*}{ Delegation } & [ABLP93] $\S 5-\S 6.1$ \\
\hline & Also: [LABW92] §7, [HK00] §4.6 \\
\hline Interprocess Communication & [LABW92] §8 \\
\hline \multirow[t]{2}{*}{ Access-Control Decisions } & [ABLP93] $\$ 6.2$ \\
\hline & Also: [LABW92] §9 \\
\hline Reasoning about Credentials and & [WABL94] $\S 1-\S 4.3$ \\
\hline \multicolumn{2}{|l|}{ Certificates } \\
\hline Extensions to the Logic & {$[\mathrm{HK} 00] \S 6$} \\
\hline
\end{tabular}

\subsection{Assessment Methods}

We used Socratic-style question-driven lectures as one means for formative assessment. For example, when introductory concepts on RBAC were presented, students were called upon to answer questions related to the definitions, properties, or applications of RBAC. To ensure the participation of all students and to randomize the order in which they are called, names are drawn from a deck of cards. Students are allowed to say "I don't know." There is no attempt to intimidate; rather, the intent is to provoke a thoughtful response. Generally speaking, if three successive students are unable to answer a question, the instructor can reasonably conclude that the class is unclear about the current topic. At that point, the instructor can decide whether more time or a different approach is needed. Similarly, if students are able to answer the questions, they have feedback that they answered the questions correctly. Students who couldn't answer the questions know that they need to study those particular topics more.

Summative assessment is accomplished through four open-book, opennotes exams. Students receive written solutions as they turn in their exams. The questions are formulated with the educational outcomes at the various levels of knowledge previously described (comprehension, application, analysis, synthesis, and evaluation). While the course emphasizes the use of formal analysis, more weight is typically given to questions that ask students to set up the formal assumptions, definitions, and goals when given an informal problem description. 


\subsection{Observations}

Fundamentals The first exam occurred four weeks into the course. Two questions dealt specifically with comprehending cryptographic algorithms (DES and RSA): given particular inputs, students had to compute the outputs. Little class time was devoted to these topics, and the average grades on these questions (out of eighteen students) were $71 \%$ and $82 \%$. At the levels of application, analysis, and synthesis, students were asked to model cryptographic algorithms in schemes such as electronic code book and cipher block chaining. Here, the results were less satisfying. For example, students were asked to model ECB encryption as a recursive function (where the particular encryption function and key are parameters) and prove that ECB inverts itself. In this case, the average score was 50\%. However, the distribution of grades was bimodal: $25 \%$ of the students received full or close to full credit, $25 \%$ received no credit, and the remaining students received between $20 \%$ and $70 \%$, with most of these students getting more than $50 \%$. All of the students who received no credit had failed to take the prerequisite course in predicate calculus.

BAN Logic and Role-Based Access Control The BAN material was also assessed in the first exam, at the application, analysis, and synthesis levels. Specifically, one question at the analytical level asked for a proof that, in the context of the X.509 protocol, one principal believed that another principal believed in a particular statement. The initial assumptions about keys and nonces were given. The average for this question was $76 \%$, with seven students getting $100 \%$ and one student getting $0 \%$. Another question asked students to consider how beliefs would change if X.509 timestamps could not be checked accurately, and to justify their answers using the BAN logic. The average for this question was $70 \%$ : ten students got $100 \%$, and three students got $0 \%$.

RBAC was assessed in the second exam, which was given after seven weeks. By this time student enrollment had dropped from eighteen to fifteen. RBAC was assessed at the levels of application, analysis, and synthesis. Students were shown an organizational chart of company roles and asked to formally prove or disprove mutual exclusivity of various roles. The average on this problem was $66 \%$, but the distribution was again bimodal. Five students earned $100 \%$, six students earned between $70 \%$ and $95 \%$, one student received $20 \%$, and the remaining three students received $0 \%$.

Authentication, Delegation, and Access Control The underlying semantics of the principal calculus of Abadi and colleagues [LABW92, ABLP93, HK00] is based on Kripke structures. A Kripke structure comprises a set $W$ of possible worlds; an interpretation function $I$, which maps each propositional variable to a subset of $W$; and an interpretation 
function $J$, which maps each principal name to a binary relation over $W$. Intuitively, $I(p)$ is the set of worlds in which the propositional variable $p$ is true, and $J(A)$ is the accessibility relation for principal $A$ : if $\left(w, w^{\prime}\right)$ is in $J(A)$, then principal $A$ cannot distinguish between worlds $w$ and $w^{\prime}$.

Questions on the second and third exam focused on assessing the students' understanding at the comprehension and analysis levels. Specifically, they were given a particular Kripke structure and asked to evaluate the beliefs of principals in various worlds. The average grade on Kripke structures in the second exam was $58 \%$, with three students receiving $100 \%$ and four students receiving $25 \%$ or less. These grades improved on the third exam to an average of $65 \%$ with five students receiving $100 \%$, one student receiving $93 \%$, and the remaining students receiving between $27 \%$ and $53 \%$.

Students were also asked on both the second and third exams to prove an axiom of the calculus (e.g., "if $(A \wedge B)$ says $\mathrm{s}$, then $A$ says $\mathrm{s}$ and $B$ says $\mathrm{s}$ "). The average grade for this question on the second exam was $48 \%$, with two students receiving $100 \%$ and three students receiving $0 \%$. On the third exam, grades improved to an average of $60 \%$, with five students receiving $100 \%$, one student receiving $80 \%$, one student receiving $0 \%$, and the rest receiving between $20 \%$ and $60 \%$.

The fourth and final exam (given to fourteen students after fourteen weeks of class) focused on reasoning about certificates, delegations, and authority and on proving properties of credentials used in the Taos operating system [WABL94]. This time, students were presented with a client/server system where the server receives a message " $C_{\text {Alice }}$ says $R Q$ " within the context of boot, delegation, and channel certificates. The students first had to set up a theory whereby the server could conclude that ((Machine as OS) for Alice) says $R Q$, and then they had to carry out a formal proof to justify their conclusions. This problem was very similar to the extended example of [LABW92]. The average score on these questions was $67 \%$, with seven students scoring $80 \%$ and above (two at $100 \%$, one at $97 \%$, and four at $80 \%$ ), and the remaining seven students with scores from $33 \%$ to $60 \%$.

\subsection{Adjustments}

This course has evolved much over the last six years. In this latest offering, we were interested in seeing how far and how deeply our students could learn to use the principal calculus of Abadi and colleagues. We were pleasantly surprised. The mid-course corrections we made were based on our observation that many students were not facile with the use of discrete mathematics (sets, relations, algebraic properties). This observation caused us to devote more time to reinforcing discrete mathematical notions at the 
expense of reasoning about additional protocols, such as electronic banking protocols. In the future, we plan on rectifying the lack of facility with discrete math by incorporating more applications such as RBAC and modal logic into the predicate-calculus course that is a prerequisite for this course.

Overall, the students were satisfied with the course, although some would have preferred less theory and more applications. In our view, limiting the time spent on cryptographic algorithms to devote time to the authentication logic was well worth it: students gained knowledge that enables them to think rigorously about authentication, certificates, delegation, and access control.

\section{CONCLUSIONS}

We have described two courses that illustrate out efforts to introduce rigorous foundations for assurance into an IA curriculum. Specifically, we have presented not only the material that we cover, but also how we assess students' mastery of that material and how they are doing. The statistics that we have provided are not by themselves statistically significant. However, we believe they shed light both on how we are doing so far and on what is possible in a Master's-level IA curriculum. Furthermore, students have responded positively to these courses: in fact, in each of the past two years, undergraduates have expressed interest in our Master's CASSA program as a result of analyzing security protocols in the concurrency course.

Based on our experiences, we advocate using an outcomes-based approach to develop courses and curricula. We are not assessment experts, but rather users who have found value in adopting practices put forward by experts in the field of Higher Education. We have tried to demonstrate that the process does not have to be painful or cumbersome. Even a lightweight approach as in CIS 632 offers a lot of benefits. A course's educational outcomes are suggestive of the appropriate assessment techniques, and both outcomes and assessment are in the instructor's control. Another advantage of writing outcomes is that information is communicated more precisely, both to students and to other faculty. Because we have thought about educational outcomes, several faculty across several courses have developed consensus regarding our Computing Engineering and Computer Science Master's programs. We are moving towards a common core for these two programs that includes logic, functional programming, and concurrency. This common core would help prepare students for the two courses discussed here, as well as for the rest of our Master's programs. 


\section{ACKNOWLEDGMENTS}

This work is partially supported by the National Science Foundation under Grant Number DUE-0241856, as well as by the Information Assurance program of the New York State Center for Advanced Technology in Computer Applications and Software Engineering (CASE).

\section{REFERENCES}

[ABLP93] Martin Abadi, Michael Burrows, Butler Lampson, and Gordon Plotkin. A calculus for access control in distributed systems. ACM Transactions on Programming Languages and Systems, 15(4):706-734, September 1993.

[BAN90] Michael Burrows, Martin Abadi, and Roger Needham. A logic of authentication. Technical report, SRC Research Report 39, Systems Research Center, Digital Equipment Corporation, Palo Alto, CA, 1990.

[Blo74] Benjamin S. Bloom. The Taxonomy of Educational Objectives: Affective and Cognitive Domains. David McKay, New York, 1974.

[CPS93] Rance Cleaveland, Joachim Parrow, and Bernhard Steffen. The Concurrency Workbench: a semantic-based tool for the verification of concurrent systems. ACM Transactions on Programming Languages and Systems, 15:36-72, 1993.

[Dia98] Robert M. Diamond. Designing \& Assessing Courses \& Curricula: A Practical Guide. Jossey-Boss, revised edition, 1998.

[FBK99] David F. Ferraiolo, John F. Barkley, and D. Richard Kuhn. A role-based access control model and reference implementation within a corporate intranet. ACM Transactions on Information and Systems Security, 2(1):34-64, February 1999.

[FK92] David Ferraiolo and D. Richard Kuhn. Role based access control. In Proceedings of 15th Annual Conference on National Computer Security, pages 554-563, Gaithersburg, MD, 1992. National Institute of Standards and Technology.

[For97] Formal Systems (Europe) Ltd, Oxford. Failures-Divergence Refinement: FDR2 User Manual, October 1997.

[For98] Formal Systems (Europe) Ltd, Oxford. Process Behaviour Explorer: ProBE User Manual, March 1998.

[FSG+00] David F. Ferraiolo, Ravi Sandhu, Serban Gavrila, D. Richard Kuhn, and Ramaswamy Chandramouli. A proposed standard for role-based access control. Technical report, National Institute of Standards and Technology, December 2000.

[HK00] Jon Howell and David Kotz. A formal semantics for spki. Technical Report TR 2000-363, Dept. of Computer Science, Dartmouth College, Hanover, NH, March 2000.

[Hoa85] C.A.R. Hoare. Communicating Sequential Processes. Series in Computer Science. Prentice Hall, London, 1985.

[LABW92] Butler Lampson, Martin Abadi, Michael Burrows, and Edward Wobber. Authentication in distributed systems: Theory and practice. ACM Transactions on Computer Systems, 10(4):265-310, November 1992.

[Lam71] Butler Lampson. Protection. In Proceedings of the 5th Princeton Conference on Information Sciences and Systems, Princeton, NJ, 1971.

[Low96] Gavin Lowe. Breaking and fixing the Needham-Schroeder public-key protocol using FDR. Software Concepts and Tools, 17:93-102, 1996. 
[Mil80] Robin Milner. A Calculus of Communicating Systems, volume 92 of Lecture Notes in Computer Science. Springer-Verlag, 1980.

[OC02] Susan Older and Shiu-Kai Chin. Building a rigorous foundation for assurance into information assurance education. In Proceedings of 6th National Colloquium for Information Systems Security Education, volume 1. George Washington University Journal of Information Security, 2002.

[Ros98] A.W. Roscoe. The Theory and Practice of Concurrency. Series in Computer Science. Prentice Hall, London, 1998.

[Sch00] Steve Schneider. Concurrent and Real-Time Systems: The CSP Approach. John Wiley \& Sons, 2000.

[SS75] Jerome Saltzer and Michael Schroeder. The protection of information in computer systems. Proceedings IEEE, 63(9):1278-1308, September 1975.

[Sta99] William Stallings. Cryptography and Network Security, Second Edition. Prentice-Hall, 1999.

[Syr98] Syracuse University Department of Electrical Engineering and Computer Science. Developing curricula to meet the needs of the next millenium: Preliminary report of the EECS Curriculum \& Course Development Committee, 1998.

[WABL94] Edward Wobber, Martin Abadi, Michael Burrows, and Butler Lampson. Authentication in the Taos operating system. ACM Transactions on Computer Systems, 12(1):3-32, February 1994. 